\title{
Is this Digital Resilience? Insights from Adaptation and Exaptation of a Cyber-Physical- Social System
}

\author{
Sindisiwe Magutshwa \\ University of Agder \\ sindisiwe.magutshwa@uia.no
}

\author{
Jaziar Radianti \\ University of Agder \\ jaziar.radianti@uia.no
}

\begin{abstract}
This paper is based on a qualitative case study that explores the adaptation and customisation of a Cyber Physical Social System (CPSS)-based patient monitoring solution for use during Covid19 in the Norwegian health sector. The study seeks to answer the following research questions: 1) what are the preconditions that enable the adaptive use of a CPSS in crisis response efforts? 2) what are the contributions of the adaptive use of technology in the building of digital resilience in a health organisation? The study identifies five main themes that emerge as enabling factors forming a basis for the preconditions to adaptive use of the CPSS. We conclude with a discussion on the practical and theoretical implications of this research and how it contributes to crisis management and digital resilience theory.
\end{abstract}

Keywords: pandemic response, cyber physical social system, digital resilience, adaptation, exaptation

\section{Introduction}

In recent years, Information and Communication Technologies (ICTs) have emerged as key elements in the strengthening of societal resilience in times of crises. Resilience primarily diminishes the shock value through the expansion of the capacity to adapt to uncertainty [1]. A current, far-reaching crisis that has come with great uncertainty and emphasizes the need for resilience through use of ICT and digital alternatives is the Covid19 pandemic. People and organizations across various sectors have pursued numerous health and operations principles, leading to different outcomes.

In the health sector, we see how digital health technologies have been centralized, and integrated into coordinated strategy and pandemic response efforts [2]. This application of digital technologies in different aspects of Covid19 planning and response is now an important area of Information Systems and Technology research [3]. These digital technology applications have been studied and linked to the building of digital resilience of people and organisations. For example, how digitally resilient organisations are better equipped to adapt to uncertainty and change using their technological channels and resources [4] .

Of course, the application of digital solutions in the health sector is not a new practice. Countries such as Norway have systematically introduced digitalised healthcare service for over 10 years and possess varied experience on the affordances and capabilities that health technologies provide [5]. However, the farranging implications of the Covid19 crisis, such as mandatory social distancing, and capacity constraints in hospitals, give rise to a rather unique set of circumstances that have caused a shift in how these health technologies should be deployed and managed.

An instance of this is in personalised healthcare, where we see the continuous usage of various sensors and digital technologies to capture the biomedical and clinical data from patients treated at home, allowing remote monitoring. Using remote sensing techniques, these technologies have complemented health personnel in varying degrees through the virtual care platforms that include video conferencing and digital patient monitoring. The systems are designed to be a secure intermediary between patients and health personnel and emulate typical patient monitoring practice. In terms of Information Systems (IS) research, such system architectures are conceptualized as Cyber-PhysicalSocial system (CPSS). In these systems, humans work closely alongside sensors, enabled devices to complete processes and operations. The CPSS ecosystem creates avenues of the possibility for collaboration between software, hardware, and social components across a wide range of modalities.

Theoretically, systems within the CPSS paradigm are reconfigurable, and known to advance capability, adaptability, scalability, and resilience [6]. A study focusing on understanding the role of such technology in digital resilience building in a health organisation is timeous[7]. While the benefits of using CPSS technologies in healthcare are well documented, there are also uncertainties, risks, and threats associated with their usage. IS and Computer Science literature is rich in studies that investigate the CPSS risks in terms of the technical aspects. However, due to the 'social' nature of this technology, the non-technical aspects of CPSS must 
also be understood and equally scrutinized as the technical dimensions.

This paper is based on a qualitative case study that explores the adaptation and customisation of a CPSS for use in Covid19 patient monitoring in the Norwegian health sector in the Fundi region, an anonymised name of the case study location. This study has the potential to provide practical insights into the enabling factors and attributes of the CPSS tool and its usage that foster adaptive use in pandemic response efforts. The paper addresses the following research questions:

- RQ1: What are the preconditions that enable the adaptive use of a CPSS in crisis response efforts?

- RQ2: What is the contribution of the adaptive use of technology in the building of digital resilience in a health organisation?

The contributions of this study lie in efforts to build a body of knowledge concerning the adaptive use of CPSS technology in health crisis response. This study also contributes to theory building on digital resilience building in organizations, which will extend current understanding of the resilience concept in health crisis response, and crisis management in general. The paper is organized into seven sections. Section 1 introduces the goal, research question and contribution of this research. Section 2 is a review on related works, while section 3 presents the case study. In Section 4 we explain the methodology conducted in this research. Section 5 presents the results from the interview, while the discussion and implications of the results are presented in Section 6. Section 7 concludes the paper and addresses the limitations of this study.

\section{Related Works}

This section provides an overview of two areas of research in the literature. Firstly, the overview of how CPSS are progressively implemented in critical sectors, with specific focus on the health sector. Secondly on the use and contribution of ICT to the digital resilience building to withstand the challenges emanating from crisis. The first literature selection is based on highly cited publications related to CPSS, details application domains, and deployments of CPSS in engineering, computer science and information systems. The second analysis is based on a set of recent and relevant literature discussing the application of ICT in crisis settings, in some cases Covid19, and how this ICT technology enables a digital resilience strategy to cope with system shock caused by the pandemic and other crises.

The CPSS paradigm is a somewhat new interdisciplinary concept, whose origins can be traced back to the widely known cyber-physical systems (CPS). CPSSs are defined as engineered systems that are built from a seamless integration of computational algorithms, physical, and social components $[6,8]$. The systems connect nature, cyber-space, and society with specified rules, typically harvesting data from the physical environment through sensor technologies, further including human actors that are a part of the system and possess their own "cognition, preferences, motivation, and behaviour" [7]. In the literature, CPSS are referred to with a variety of research discipline specific terms such as Socio-Cyber-Physical Systems, Cyber-Physical-Human System [9]. The terms emphasize the socio-technical nature of the systems. A recent trend has seen the implementation of CPSS in critical sectors, in application domains such as personalised healthcare, emergency response, and smart manufacturing [5]. In healthcare, CPSS are also often referred to as Medical Cyber-Physical Systems (MCPS). The systems are integrated into missioncritical process, where patients, clinicians, smart medical devices, embedded software, and remote networking capabilities work collaboratively to meet vital health service goals [10].

The Covid19 pandemic has triggered the emergence of such digital technology initiatives in higher numbers. We have seen innovative and improvised use of CPSS technologies as tools for pandemic preparedness and response in various domains. These varied applications have enabled forecasting of resource allocation based on patient survivability data, hospital capacity monitoring, and tracking of infection rates. Kohn [4] asserts that ISsupported adjustment to disruption, where people overcome technical, and motivational challenges to maintain a level of productivity in the same level as prior to disruption are considered digitally resilient. While the pandemic has accentuated the beneficial aspects of digital technologies and how they enhance resilience in healthcare, the use of CPSS in the health sector demands a level of reliability, predictability, and safety. These ecosystems are not without complications. They present with many unknown threats and risks, triggered by combined technical, operational, and social factors. Patient data protection and privacy are the primary concerns when dealing with CPSS. In addition, the non-technical aspects and human-induced risk have not been emphasized in the literature and must be addressed in research [11].

This surge of various technology-based solutions in response to crisis has led researchers to further emphasize the importance of research on how the application of digital technologies in crisis response efforts builds digital resilience in organisations[12]. Digital resilience is described as "the phenomena of designing, deploying, and using information systems to quickly recover from or adjust to major disruptions from external shocks" [13]. Scholars have suggested several 
dimensions of resilience in different domains such as in crisis management and disaster recovery, cybersecurity, business continuity and information systems[14, 15]. In [16], the authors conceptualise a resilience for the IS research domain that the authors in [12] refer to as an IS resilience. The authors in [12] conceptualise digital resilience as a phenomenon enabling the resilience of the supra-system, i.e., the resilience of the organisation using the IS. This highlights a shift in research approach, from analysing resilience as just a system property [13], to an ecosystem centric approach, factoring in the immediate environment in which the system operates [12].

In this paper we assume an ecosystem perspective and define digital resilience as the "capacity of individuals, organisations, and society to make effective use of digital technology to prevent, anticipate, absorb, and adapt to major challenges and to continuously evolve and transform in a productive and sustainable manner" [17-19]. This perspective aligns to disaster resilience approaches[20], where it is important to identify not only how the system is resilient[21], but what it is resilient to and how it exhibits that resilience [22]. We assume this stance for two reasons, firstly, the nature of the crisis - Covid19, has impacted organisations on multiple fronts, and emphasizes the need for investigation of a resilience that emerges as a direct consequence of the use of digital technologies such as CPSS in crisis response efforts. Secondly, we focus on CPSS- type information systems, due to the mission-critical role they progressively play in critical societal processes. The qualitative dissimilarity of CPSS components (social, physical, and computational) highlights a need for the investigation of resilience within these systems from an approach that considers not only the technical aspects but the full system composition.

The literature analysis highlights how IS research does not provide solid frameworks or models leading to understanding digital resilience in IS, instead it offers "a rather shallow and narrow understanding of resilience" [12, 16]. Majority studies focus on theoretical conceptualisation of digital resilience, while limited studies analyse the empirical aspects such as dimension indicators. It is a challenge to attempt to measure digital resilience because it is a theoretical concept. However, the assumption of an ecosystem perspective facilitates process thinking and this fosters the possibility of empirical observations of multidimensional indicators for digital resilience [7, 22]. Organisations implement innovative, collaborative, and secure approaches to their strategic decision-making in times of crisis. These are well documented in research [23]. However, there is value in revisiting these dimensions within the context of adaptive use of a digital technology[24]. The use of existing digital technologies in crisis response efforts may ultimately contribute to the building of digital resilience in organisations and the analysis of the strategic and technical processes could reveal such connections [6]. The following section details the case study and how the study is designed and executed.

\section{Case Description}

We used the Fundi region in Norway as the case study. The region has been a part of the National Welfare Technology program since 2013, with multiple digital healthcare projects targeting chronically ill (e.g., diabetes), and elderly patients. The 'digital-follow-up' analysed in this study, is based on an earlier technology, initially designed in partnership with Org-X, the technology vendor for use in the welfare technology program. In the initial patient protocol, the patient was allocated a kit, comprising a smart tablet, condition dependent biosensors, and medical measuring equipment for home use.

From a system architecture perspective, the patient and all the infrastructure can be referred to as a CPSS. The body vitals were captured with the help of the patient, transmitted through a Wi-Fi connection, and then monitored remotely via telemedicine centres (TMS) in the municipal health services. In the case of complications and other emergency issues, the real time support and follow up is also enabled through video, messaging, or telephonic calls. The service was tested in hospital environments, and at a later stage for mental health patients.

When the pandemic hit Norway in February 2020, upon the realisation of the possibility of the hospitals being flooded with patients, a project group with experience in digital follow-up (CPSS tool) in the Fundi region made an assessment and decided to adapt this digital follow up tool to anticipate the likely overcapacity issue of the hospitals in the region. However, this was an intuitive decision, based on the multiple years of experience with digital home followup. The update was done in cooperation with the same technology vendor $\mathrm{Org}-\mathrm{X}$. Over a period of $2-3$ weeks, a newly introduced Covid19 module was developed with a deployment protocol that allowed the patients to 'bring their own device - (BYOD)'. It was believed that the adaptive use of existing digital patient monitoring technology to remotely monitor and consult with Covid19 patients would provide a buffer to and enhance the capacity of the health service and potentially contribute significantly to crisis response efforts. This study follows this recreation process of the CPSS monitoring tools and monitoring protocols for Covid19 patients. 


\section{Methodology}

This research is an exploratory case study in essence[25]. To address our research questions posed in the Introduction section, we used combined qualitative techniques for collecting the necessary empirical data for analysis. Figure 1 is an illustration providing oversight of the research procedure.

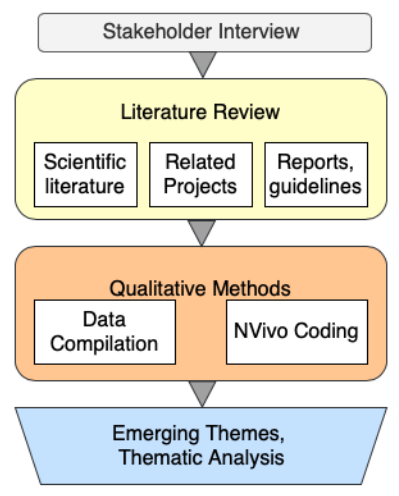

Figure 1: Overview of research methodology

\subsection{Research Procedure}

Given the exploratory nature of this study, we began with implementing stakeholder interviews, as explained further in Section 4.2. This empirical study follows the progressions in the development of the Covid19 module, from strategic decision making, through to the technology development, implementation, and evaluation. The relevant stakeholders that were involved in this repurposing process of the welfare technology to Covid-19 patient monitoring were identified. The relevant informants included clinicians, technology vendors, and managerial personnel. We then conducted a preliminary literature review for identification of research gaps and thematic corroboration. We selected recent, highly cited, scientific publications that identify the prevailing understanding of the key concepts of digital resilience and CPSS ecosystems. The activities and findings related to the literature analysis are mainly discussed in the 'Related Works' section of this paper. We further collated and analysed the secondary data sources from related welfare technology projects, reports, and guidelines. These are primarily organisational documents linked to usage history of the digital tool in the organisation and experience reports following use of the tool for Covid19 patients.

Data was compiled and coded using NVivo, a software tool used for qualitative data coding and categorisation, and for maintaining the consistencies of the results. The NVivo coding process also helped the analysis process such as identifying the emerging themes and conducting thematic analysis, such as the identification of the enabling factors leading to the successful adaptation of the digital tool.

\subsection{Data Collection and Interview Process}

The data collection was carried out in two phases. The first phase was done in July 2020, focusing on understanding the early version of the digital follow up tool and how it was adapted for monitoring Covid19 patient. We began with a live demonstration with Org$\mathrm{X}$, the technology vendors. In addition, the data collection and analysis of records, documents, meeting minutes, and reports supplied by the Fundi region were done at this stage.

Table 1: Study informant profiles

\begin{tabular}{|c|c|c|}
\hline$\div$ & Position & Organization \\
\hline Inf-1 & $\begin{array}{l}\text { Head of Digital and Enterprise } \\
\text { Services }\end{array}$ & Org-X \\
\hline Inf-2 & Digital Solution Lead & Org-X \\
\hline Inf-3 & $\begin{array}{l}\text { Head of Research \& Medical } \\
\text { Doctor }\end{array}$ & $\begin{array}{l}\text { Fundi } \\
\text { Municipality }\end{array}$ \\
\hline Inf-4 & $\begin{array}{l}\text { National Welfare Technology } \\
\text { Program Manager \& ex } \\
\text { Rescue Medic }\end{array}$ & Fundi Region \\
\hline Inf-5 & $\begin{array}{l}\text { eHealth Research Innovation } \\
\text { Manager }\end{array}$ & $\begin{array}{l}\text { Fundi } \\
\text { Municipality }\end{array}$ \\
\hline Inf-6 & eHealth Advisor & Fundi Hospital \\
\hline Inf-7 & Nurse & Fundi Hospital \\
\hline Inf-8 & $\begin{array}{l}\text { Project Lead - Digital follow- } \\
\text { up (Design) \& ex Nurse }\end{array}$ & Fundi Region \\
\hline Inf-9 & General Practitioner & $\begin{array}{l}\text { Fundi } \\
\text { Municipality }\end{array}$ \\
\hline Inf-10 & $\begin{array}{l}\text { Project Lead - Digital follow- } \\
\text { up (Security) }\end{array}$ & Fundi Region \\
\hline Inf-11 & $\begin{array}{l}\text { Welfare Technology } \\
\text { Distribution Lead }\end{array}$ & Fundi Region \\
\hline
\end{tabular}

Follow up interviews were then conducted with Informants 1 and 2 (see Table 1). These interviews provided details on the technical background of the CPSS solution, and the necessary adjustments required for use in Covid19 patient monitoring. A second data collection cycle was done in the period between November 2020 and January 2021. This phase was geared towards understanding the experiences linked to the implementation and evaluation of the Covid19 module of the solution. Due to changing Covid19 restrictions in the country, the interview format was blended, some were implemented digitally using the Zoom platform, and also in person, when possible. All but one of the interviews were conducted in English, so there was also a process of translation from Norwegian to English in addition to the transcription process. The total time used for the interviews were 24.5 hours and the data was managed and processed on NVivo. 


\subsection{Data Analysis}

It is nearly impossible to separate the interview and analysis phases of the research. The processes were often entangled and characterised by multiple iterations between the data and literature. In [25], the authors propose a systematised approach to inductive research in which the interview data is categorised in three phases. Inspired by this, the data analysis was completed in three iterative analytic phases. In phase 1 , we identified the descriptive keywords and text extracted from the interview transcripts. And because we intended to use the 'ecosystem perspective' at this stage, it was necessary to identify the involved subsystems within the health organisation, this would be a safeguard for ensuring representation from all involved subsystems (details on this are further discussed in section 6). We traced the legacy systems, identifying the system roots, and what was in existence prior to the shock. The second phase involved the logical sequencing of the steps and procedures as they were executed throughout the project and identification of emerging themes. The third phase was sense making, a combination of conceptually mapping the themes identified in phase 2 and finding a deepened understanding of the empirical observations through a theoretical lens. The results were analysed following an interpretive stance, focusing on the identification of enabling factors in the steps taken during the design, development, implementation, and evaluation of Covid19 patient monitoring module.

\section{Results}

This section reports the findings of the study. It is arranged in a narrative style, providing insight into the context, actions, and general experience from the informants' perspective. The results are organized into five main themes: Covid19 Response timeline, Knowledge and Skill Gaps, Innovation and Digital Alternatives, Leadership and Collaborations, and Risk and Security.

\subsection{Covid19 Response Timeline}

At the onset of active cases (February $21^{\text {st }}, 2020$ ) in Norway, the crisis management protocols in the Fundi region had already been mobilised in line with national guidelines. Considering the uncertainty, a suitability assessment of existing infectious disease management and response procedures and protocols had to be done. The study informants expressed that they realised that there was inadequacy of existing prescribed strategy and preparation as stipulated in the crisis management plan for this nature of an infectious disease. It was at this point that the decision to develop a Covid19 module for digital monitoring was made. As mentioned in Section 3 , the motivating factor behind the decision to develop a Covid19 module for the digital monitoring was to buffer the hospital system from floods of patients and limit physical contact between healthcare professionals and infected patients. On March $11^{\text {th }}$, a day sooner than national government, the Fundi region called for residents to go into lockdown. Based on the timeline, the development work on the digital tool was already underway at this stage, and by March $19^{\text {th }}$, the solution was available for use. The following subsections are a summarized description of the key details of the project relevant to this study's objectives.

\subsection{Knowledge and Skills Gap}

A key hurdle identified at the very beginning of the project has been the limited availability of knowledge on the disease. Competence building was initiated well in advance, prioritising crisis response strategy, patient care, and health personnel safety. A great deal of resource/personnel allocation, and structuring was required, to ensure representation of all stakeholders. While the digital tool had been in use in the health service for years, it had only been used for widely known, well researched conditions such as diabetes, heart failure, and pulmonary disease. Monitoring algorithms had been developed for these conditions based on years in medical research. Inf-3 recalled: "the problem with COVID-19 was we didn't know all the symptoms... we had to read as much as we could and get a specialist to come in and try to build the algorithm and further develop the algorithm as we went on". The development of the algorithm was a key component of the Covid19 module, and despite the knowledge gap, the team had to ensure it would capture all essential aspects of the disease. A team of medical doctors including a pulmonary specialist was assigned the task of developing the algorithm. Further information was sourced on an ongoing basis from organizations such as the World Health Organization (WHO), and the Norwegian Health Directorate. With content and protocol covered, the technical development was then headed by Org-X, a long-standing partner of region. Inf1 said: "It was a hectic time and stressful because we worked a lot, maybe around 70 hours on some of the weeks". This was by and large a result of a series of further changes required for the Covid19 module beyond just the algorithm (these are discussed later in the next section). The presence of predefined organizational structures and partnerships with institutions such as Fundi hospital, and Org-X were key contributors to the hastened progression in this phase of the project. 


\subsection{Innovation and Digital Alternatives}

Further significant changes were made to the system to customise it for Covid19 patient use. There was a marked difference in patient demographic, from elderly, chronically ill patients to practically anyone. For one, the system deployment strategy needed to be revised, to accommodate greater numbers and improved accessibility. The previous protocol had involved the provision of tablets, and this was not economically feasible and had to be reviewed. This was where the BYOD came in. Patient registration was migrated to a web interface and a downloadable application available in the Google and Apple stores. Org-X had to make internal changes to accommodate some of these emerging requirements. Inf-2 recalled: "the web development, this is normally not something we do within Org-X. Normally, we provide an all out of the box products that maybe need some kind of installation or configuration". This meant patients could create profiles, self-register and continue to do their own daily assessments. The self-assessment was a patient questionnaire in the application, developed with the help of medical personnel which would be used to determine the severity of the patient's condition. This was a major change that introduced a wide range of security and technical complications. Migration from a secure, password locked tablet also meant user identification and authentication became priority.

There had not been integration to the population registry before, but for the new protocol, this became a prerequisite. A separate installation was ordered for the authentication, and it was integrated with the Norwegian Population Registry through an electronic personal identification system called BankID. This automated registration and self-assessment was particularly useful for patients in the early phase of infection. Inf-4 shared: "My hypothesis was if you don't know how they're doing (before hospital admission), you don't know how to prepare the (health) system.". Inf-8 shared about the value in end-stage/post-infection monitoring: "it might be of interest to follow up long term effects of COVID19 for those who have only partly recovered." . This approach enabled continuous refinement of the patient registration questionnaire and follow up algorithm as new information became available. Also, there was added value, an opportunity to harvest data on the longterm patient recovery patterns. Once the system was ready, a round of testing was done by Org-X during the development phase. It was an improvised testing regime, and Inf-2 described it as follows: "we did a lot of testing, just in a different way. on healthcare providers, ourselves, every spouse in the organization was registered as a test guide... even my mother-inlaw". Pilot testing and implementation then followed.

\subsection{Leadership and Collaboration}

At this point it is important to note that the Fundi region acts as the administrative body for several municipalities that are located within the region. While the project team responsible for the development work of the Covid19 module operated at a regional level, when it came to the decision to implement the system, it had to be made at a municipal level. Inf-11) recalled: "we contacted the municipalities we already had collaboration with, to see if they could test it out and some of them did and some of them didn't." The municipalities were given the prerogative to further customise the roll out strategy based on their individual needs. This study included two different municipalities. The first, named Goodwill (pseudonym), had concerns on the use of the TMS centres for the data monitoring, and preferred that the municipal general practitioners (GPs) incorporate the tool into their practice. The project team set up this course of service and did another round of testing, located in a GPs practice following six patients that had been ill between March and May but recovered. The leadership of Goodwill municipality eventually opted out of the solution, citing the need for further technical development, and training. At another pilot test, at the Fundi Hospital, ten Covid19 patients who were at various stages of infection were used as test subjects. Input was given on usability and content and used to improve the self-assessment questionnaire, algorithm, and application user interface.

The hospital opted to use the solution to monitor end stage infection patients, that could be given early discharge and sent home to complete recovery with the monitoring kits. The solution was ready for use but as it were, infection numbers in the first wave (February to June 2020) of the disease were relatively low in the Fundi Region and interest in the solution waned. The project team used the intermission to take a break for reflection. The time allowed for extensive assessment of the system, with in depth consultation of experts. Inf-4 recalls: "I had some discussions with some friends in a pretty big international network - out of Italy and out of Asia, US region, to see how they are doing it, application user managers and designers". The feedback informed a learning process at this stage that was used in further refinement of the system. In November 2020, reports emerged of an outbreak in the Fundi region's Shaka municipality (pseudonym). The municipality had prior experience with the digital follow-up and the project team approached the municipality to propose use of the solution for the outbreak. The leadership opted in, with no requirement for any further changes to the roll out protocol. The application was used to monitor 65 patients and following its usage, an evaluation process followed. 
Based on a report that captured the user sentiments of patients through a post treatment survey revealed that patients found the solution to be "brilliant and very easy to understand and use". Most respondents gave positive feedback when prompted if they had felt increased security due to availability of the application.

\subsection{Risk and Security}

The nature of the Covid19 crisis and the chosen response strategy of the Fundi Region required the consideration of risk and security from a tactical and a strategic perspective. The project manager (Inf-4) said: "My nightmare was a headline in the papers about a patient data leakage. Because we were going from a few patients on an iPad tablet format working on $4 \mathrm{~g}$, where the risk is low, it's completely different stuff when you're on your own device, on iOS, Android, it's on Wi-Fi". Tactically, there needed to be technical changes made because of the decision to deploy the system on a larger scale through an application and a web interface. The change in user demographic had risk and security implications because while an elderly, eighty-year-old user on a locked tablet was unlikely to be malicious. The possibility of younger people, with greater technical skills and harmful intent on Wi-Fi was a threat that needed to be addressed. Steps to mitigate potential risk at physical infrastructure level were taken. Inf-2 recalled: "risk assessment? we really did that, together with the Fundi region and Goodwill municipality. We had consultants to perform the penetration tests of the technical solution to make sure it would secure personal data”. Two-factor authentication was introduced as one of the several steps taken to securing the application. However, further internal structures and steps were taken to safeguard against strategic risk, at the social components level [26]. Inf-10 said: "Org-X told us that it was secure, but we put together our own task force just working to see if we have all the boxes checked before we went to launch". An example of the strategic risk identified during this process was the possibility of the algorithm not capturing the deterioration of a patient. It was of utmost importance that patients receive the acceptable standard of care, and system redundancy protocols had to be decided on. The general trend was that patients would have a sudden dip on day 5. So, even if a client had been classified as 'green' by the algorithm for 4 consecutive days, on the fourth day, a clinician was to call them and confirm their condition through video or telephonic consultation, thereby verifying the system data. This was a clinical decision made to ensure patients still received optimal clinical care while at home. The following section details the analysis and discussion of the results section.

\section{Discussions and Implications}

This case was selected for two reasons, firstly, the digital monitoring tool was not designed for use in the clinical management of Covid19 patients, this was an adapted application. Secondly, the adaptive changes made to the application led to novel roll out protocols and it was one of the early applications of digital solutions used in the monitoring of Covid19 patients at various stages of infection. It is not a complex instance of technological adaptation as the tool was already in use in the health sector. However, the examination of the short- and long-term events builds a narrative that may be used to understand the suitability and effectiveness of similar solutions in the context of crisis. The choice to use an interpretive approach for this case analysis was necessitated by the need to understand the strategic decision making and adaptation of the CPSS technology from the informant's point of view. The reflections and narratives provided by the study informants in the previous section provide detailed insight into their experiences. They highlight how innovative, forward-thinking approaches and digital alternatives are required for them to generate a viable digital follow up solution for Covid19 patients. It is remarkable how the organisational leaders express an openness to 'lacking knowledge' and build capacity through collaborative leadership and network consultations. The findings make it possible to draw insights from their past experiences, extract the necessary lessons and make theoretical projections, visualizing novel applications or future possibilities for CPSS technologies in the context of crisis. The following section provides further discussion on the findings and the key outcomes and contributions of the study.

\subsection{Discussion}

Prior to delving into reflection on the Covid19 module development, a key assumption in this study is the use of the 'ecosystem' perspective. As mentioned in the 'Data Analysis' section of the paper, it is important for this study to define the health organisation ecosystem and how the different subsystems are integrated. Figure 2 is an illustration of the health organisation ecosystem. The Covid19 pandemic is the crisis domain and a pivotal shock or stressor to the health organisation. Because of crisis induced uncertainty, the crisis response strategy of the health organisation was in the short-term focused on the leveraging of the available and usable digital health technologies. It emerges that for this project digital health technology, physical healthcare, and social 
subsystems are instrumental in the generation of response efforts to the crisis in the organisation.

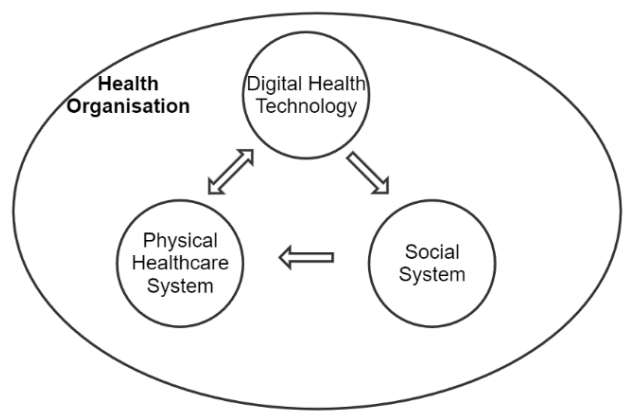

Figure 2: The health organisation ecosystem

The point of the study is to learn about how a technology module was developed to solve critical operational constraints posed by the pandemic. This proposition is consistent with other studies that emphasize how crisis results in unstable conditions that favour the search for and identification/ development of innovative solutions even with limited information on the crisis domain $[17,22]$ The first question RQ1, seeks to identify the preconditions within the health organisation that enable the adaptation of the CPSS tool for use in Covid19 patient monitoring. An 'adaptable' system is documented to have the capacity to sense, trigger, select applications rules to facilitate the necessary changes. Four main themes emerge as enabling factors in the findings, i.e., knowledge and skills gap, innovation and digital alternatives, leadership, and collaboration. They form a basis for the identification of preconditions. With this understanding and that of the health organisation ecosystem, it is possible to trace and identify these enabling factors and highlight how the CPSS digital tool possesses various traits and attributes located within the subsystems identified in Figure 2. It emerges in the data that the process of adaptation in this project was a continuous and connective process comprising multiple iterations. Because the CPSS comprises of different elements of the subsystems, they all interacted to generate a comprehensive digital monitoring solution. Table 2 summarizes the main preconditions identified in the study that led to the successful adaptive use of the CPSS technology. There are technical, social, and physical infrastructure factors that led to this outcome, and this is shown in Table 2 . The process iterations are a response to the need to continuously learn in support of the incremental innovation during the technology adaptation process. A key insight is that the technical attributes of the CPSS tool (digital infrastructure constructability and diversity) is a key enabler to the swift customisation of the technology.
Table 2: Enabling factors for technology adaptation

\begin{tabular}{|c|c|}
\hline & Preconditions for Adaptation \\
\hline $\begin{array}{l}\text { Digital } \\
\text { Health } \\
\text { Technology }\end{array}$ & $\begin{array}{l}\text { Technological/ digital infrastructure } \\
\text { constructability and diversity. } \\
\text { - System instrumenting to minimise } \\
\text { complexity and maintain possibility of } \\
\text { multiple system abstractions. }\end{array}$ \\
\hline $\begin{array}{l}\text { Social } \\
\text { System }\end{array}$ & $\begin{array}{l}\text { - Presence of human sensors and human } \\
\text { infrastructure } \\
\text { - Pre-existing interrelationships among } \\
\text { vital stakeholders }\end{array}$ \\
\hline $\begin{array}{l}\text { Physical } \\
\text { Healthcare } \\
\text { System }\end{array}$ & $\begin{array}{l}\text { - Cumulative long-term growth in health } \\
\text { technology research and development } \\
\text { - Availability of versatile staffing } \\
\text { compliment } \\
\text { - Short-term training and Capacity } \\
\text { building }\end{array}$ \\
\hline $\begin{array}{l}\text { Cognitive } \\
\text { Traits }\end{array}$ & $\begin{array}{l}\text { - Presence of boundary pushers and } \\
\text { visionaries }\end{array}$ \\
\hline $\begin{array}{l}\text { Socio- } \\
\text { economic } \\
\text { status } \\
\text { factors }\end{array}$ & $\begin{array}{l}\text { - Readily accessible stable, high-speed } \\
\text { internet connectivity } \\
\text { - User education and positive } \\
\text { psychosocial attitudes to health } \\
\text { technology }\end{array}$ \\
\hline
\end{tabular}

The digital follow-up is an embedded CPSS, integrated into a wider healthcare services network with interactions between other computational systems (patient records management) and physical human and medical systems (clinicians, doctors, patients, biomedical sensors). The CPSS is instrumentalized within the health organisation to optimize scalability, fast response time, with minimal complexity in the clinical management of Covid19 patients. The availability of human sensors and infrastructure is an instance of versatility of the system and the social layer integration. Patients are primarily a part of the system to receive healthcare, however, they also have a secondary role, that of human sensors, used to complete the monitoring process and monitor for algorithm inaccuracy. It is also important to note the socioeconomic status factors - these are directly linked to the local environment/ country. The ready availability of high-quality internet connectivity, and positive psychological attitudes to health technology by the patients is key in making the solution relevant.

\subsection{Implications: Is it Digital Resilience?}

Concerning the practical implications, by deepening the analysis, we can stratify the preconditions identified in Table 2 differently, i.e., strategy-driven preconditions and technology-driven preconditions. The technologydriven preconditions naturally highlight the key enabling attributes of the CPSS technology and how the 
nature of the technology strengthens the resilience of the health organisation. Due to the close interactions among the cyber, physical, and social subsystems, the CPSS possesses multiple mechanisms for communication, sensing entities, and end user maintenance. This is what makes the tool adaptable in technical terms. A combination of improvised research and strategic decision-making generated the innovative Covid19 module to cope with negative effects of the pandemic before effective vaccines and other viable treatment channels were available. Strategy-driven preconditions are emphasized in the self-organisation and persistence displayed by the human actors in the social and physical systems whose cognitive traits, and positive psychological attitudes inclined them towards flexibility, creativity, self-organisation, antifragility[27], and learning. Uniquely, the identification of preconditions such as socio-economic status factors reveals that the multi-layered architecture of the CPSS ecosystem enables it to contribute to not only the digital dimension of resilience but possibly to organisational and community resilience factors as well.

Recall that RQ2 asks about the contribution of the adaptive use of technology in the building of digital resilience in a health organisation. The theoretical implications of the study serve to address this RQ2. We contribute to the emerging literature on digital resilience by delving into the adaptation process and proposing insights on digital resilience building through crisisdriven innovation and adaptation of digital technologies. The themes of innovation and adaptation emerging from the data are consistent with our understanding of resilience, which we define as "the quick regaining of essential capabilities to perform critical missions during crisis and smoothly return to fully stable operations".

In this case, the decision to implement a novel clinical management methodology on Covid19 patients was wholly necessitated by the nature of the crisis. Adaptation of technology in times of crisis is highlighted as also found in the literature dealing with the digital resilience [28, 29]. However, our study is unique in terms of the fact that the digital follow up tool was initially adapted for remote monitoring of patients with well-studied diseases, but eventually secondary features prove useful for additional purposes such as the Covid19 case. In our study, this is demonstrated from the decision to use the tool on late-stage infection and recovering patients for long-term data gathering purpose. At this point the technology has not only been adapted for a new application area, but it is also further exapted. Technological exaptation is conceptualised in theory as the repurposing of traits, technologies, processes, skills, and resources for emergent uses that they were not initially designed for [30-32]. The generation of new knowledge about the disease is an exapted use of the digital monitoring tool. Adaptation and exaptation often occur in combination in crisis conditions resulting in a dynamic innovation process. It is a connective, and continuous process.

A key identifier for exaptation is that:

- the adaptation and innovation emerge without the need for a project to start from scratch, and implementable based on short-term developments to address any new requirements.

- the agility and flexibility of arrangement and governance of decision-making structures in an organisation while in crisis.

In digital resilience theory, a combination of technology and individual capacities is used to expand the support structure following a disaster or crisis by harnessing the available resources, possibilities, and opportunities [24]. However, existing literature is underdeveloped and does not identify indicators for digital resilience. This study finding provides insight into this area and shows how the Covid19 crisis creates an environmental niche that demands technological adaptation and eventually exaptation. Digital resilience is found to be less of a static state and more like a process of building capacities. This is demonstrated by how the Covid19 crisis leads to the assimilation of new knowledge and new intelligence from the emergent crisis management tools. Digital resilience is perceived as resultant from the shock/stress caused by the pandemic. Although this study is not designed to measure digital resilience, it provides insight into how it may be observed through the identification of the digital resilience dimension indicators found in this study, i.e., adaptation, innovation, and the unexplored - exaptation. This is a novel finding and a contribution to digital resilience theory.

\section{Conclusions and Limitations}

In this study we attempt to build a body of knowledge concerning adaptive use of CPSS technology in health crisis response, the strategy-driven preconditions and technology-driven preconditions leading to this phenomenon are highlighted. We also contribute to digital resilience theory, through identification of exaptation and adaptation as dimensions for indicators. The limitation of this study is threefold: Firstly, this study observed the Covid19 response in the period March 2020 - January 2021. It is likely there have been further developments following extended usage of this system and changes in the pandemic response strategy, this is not within the study scope. Secondly, digital resilience is a relatively new dimension in resilience literature and requires further operationalizations and measurements. Thirdly, this 
study is conducted in a developed country setting that satisfies all the enablers, it would be informative if further research focusing on the enabling factors of digital resilience in developing countries was

\section{References}

[1] M. Sakurai and H. Chughtai, "Resilience against crises: COVID-19 and lessons from natural disasters," European Journal of Information Systems, vol. 29, no. 5, pp. 585-594, 2020.

[2] S. Whitelaw, M. A. Mamas, E. Topol, and H. G. Van Spall, "Applications of digital technology in COVID-19 pandemic planning and response," The Lancet Digital Health, 2020.

[3] D. E. O'Leary, "Evolving information systems and technology research issues for COVID-19 and other pandemics," Journal of Organizational Computing and Electronic Commerce, vol. 30, no. 1, pp. 1-8, 2020.

[4] V. Kohn, "How the Coronavirus Pandemic Affects the Digital Resilience of Employees," PACIS 2020 Proceedings. , 2020.

[5] P. Kummervold et al., "eHealth trends in Europe 20052007: a population-based survey," Journal of medical Internet research, vol. 10, no. 4, p. e42, 2008.

[6] N. Dey, A. S. Ashour, F. Shi, S. J. Fong, and J. M. R. Tavares, "Medical cyber-physical systems: A survey," Journal of medical systems, vol. 42, no. 4, p. 74, 2018.

[7] V. Kohn, "How Employees' Digital Resilience Makes Organizations More Secure," PACIS 2020 Proceedings. 190, 2020.

[8] Y. Zhou, F. R. Yu, J. Chen, and Y. Kuo, "CyberPhysical-Social Systems: A State-of-the-Art Survey, Challenges and Opportunities," IEEE Communications Surveys \& Tutorials, vol. 22, no. 1, pp. 389-425, 2019.

[9] S. K. Sowe, E. Simmon, K. Zettsu, F. de Vaulx, and I. Bojanova, "Cyber-physical-human systems: Putting people in the loop," IT professional, vol. 18, no. 1, pp. 10-13, 2016.

[10] H. Qiu, M. Qiu, M. Liu, and G. Memmi, "Secure health data sharing for medical cyber-physical systems for the healthcare 4.0," IEEE journal of biomedical and health informatics, vol. 24, no. 9, pp. 2499-2505, 2020.

[11] S. S. Ambarkar and N. Shekokar, "Toward smart and secure IoT based healthcare system," in Internet of Things, Smart Computing and Technology: A Roadmap Ahead: Springer, 2020, pp. 283-303.

[12] M. Schemmer, D. Heinz, L. Baier, M. Vössing, and N. Kühl, "Conceptualizing Digital Resilience for AI-based Information Systems," in Proceedings of the 29th European Conference on Information Systems (ECIS), An Online AIS Conference, June 14-16, 2021, 2021.

[13] W. Fong Boh, Padmanabhan, B., and Viswanathan, S, "Call for Papers MISQ Special Issue on Digital Resilience," MIS Quarterly vol. Special Issue Call for Papers 2020.

[14] J. J. Gonzalez et al., "Stalking Resilience," Cham, 2017: Springer International Publishing, in Information Technology in Disaster Risk Reduction, pp. 31-45.

[15] J. Radianti, "Towards European Dimensions of City Resilience," Cham, 2017: Springer International Publishing, in Information Technology in Disaster Risk Reduction, pp. 104-118.

[16] R. Heeks and A. V. Ospina, "Conceptualising the link between information systems and resilience: A developing country field study," Information Systems Journal, vol. 29, no. 1, pp. 70-96, 2019. conducted. In this case, this limitation is an opportunity for future researchers with interest in the further exploration of the topic.

[17] M. Sakurai and J. Kokuryo, "Design of a resilient information system for disaster response," 35th International Conference on Information Systems: Building a Better World Through Information Systems, ICIS 2014, 2014.

[18] Y. Tim, L. Cui, and Z. Sheng, "Digital resilience: How rural communities leapfrogged into sustainable development," Information Systems Journal, vol. 31, no. 2, pp. 323-345, 2021.

[19] B. Manyena, G. O'Brien, P. O'Keefe, and J. Rose, "Disaster resilience: a bounce back or bounce forward ability?," Local Environment: The International Journal of Justice and Sustainability, vol. 16, no. 5, pp. 417-424, 2011.

[20] K. Magis, "Community resilience: An indicator of social sustainability," Society and Natural Resources, vol. 23, no. 5, pp. 401-416, 2010.

[21] A. Asadzadeh, T. Kötter, P. Salehi, and J. Birkmann, "Operationalizing a concept: The systematic review of composite indicator building for measuring community disaster resilience," International journal of disaster risk reduction, vol. 25, pp. 147-162, 2017.

[22] C. W. Zobel and M. Baghersad, "Analytically comparing disaster resilience across multiple dimensions," SocioEconomic Planning Sciences, vol. 69, p. 100678, 2020.

[23] R. A. Gonzalez, "Coordination and its ICT support in Crisis Response: confronting the information-processing view of coordination with a case study," in Proceedings of the 41st Annual Hawaii International Conference on System Sciences (HICSS 2008), 2008: IEEE, pp. 28-28.

[24] Z. Assarkhaniki, A. Rajabifard, and S. Sabri, "The conceptualisation of resilience dimensions and comprehensive quantification of the associated indicators: A systematic approach," International Journal of Disaster Risk Reduction, p. 101840, 2020.

[25] D. A. Gioia, K. G. Corley, and A. L. Hamilton, "Seeking qualitative rigor in inductive research: Notes on the Gioia methodology," Organizational research methods, vol. 16, no. 1, pp. 15-31, 2013.

[26] P. Marana et al., "Towards a resilience management guideline-Cities as a starting point for societal resilience," Sustainable Cities and Society, vol. 48, p. 101531, 2019.

[27] N. N. Taleb, Antifragile: Things that gain from disorder. Random House Incorporated, 2012.

[28] A. Rai, "Editor's comments: The COVID-19 pandemic: Building resilience with IS research," Management Information Systems Quarterly, vol. 44, no. 2, pp. iii-vii, 2020.

[29] F. F. Suarez and J. S. Montes, "An integrative perspective of organizational responses: routines, heuristics, and improvisations in a mount everest expedition," Organization Science, vol. 30, no. 3, pp. 573-599, 2019.

[30] N. Dew and S. D. Sarasvathy, "Exaptation and niche construction: behavioral insights for an evolutionary theory," Industrial and Corporate Change, vol. 25, no. 1, pp. 167-179, 2016.

[31] R. Garud, J. Gehman, and A. P. Giuliani, "Technological exaptation: A narrative approach," Industrial and Corporate Change, vol. 25, no. 1, pp. 149-166, 2016.

[32] S. J. Gould, "Exaptation: A crucial tool for an evolutionary psychology," Journal of social issues, vol. 47, no. 3, pp. 43-65, 1991. 